EPJ Web of Conferences 64, 05002 (2014)

DOI: $10.1051 /$ epjconf/ 20146405002

(C) Owned by the authors, published by EDP Sciences, 2014

\title{
MHD Simulations of Magnetized Stars in the Propeller Regime of Accretion
}

\author{
Patrick Lii ${ }^{1}$,, Marina Romanova ${ }^{1}$, and Richard Lovelace ${ }^{1,2}$ \\ ${ }^{1}$ Department of Astronomy, Cornell University, Ithaca, NY 14850 \\ ${ }^{2}$ Department of Applied and Engineering Physics, Cornell University, Ithaca, NY 14850
}

\begin{abstract}
Accreting magnetized stars may be in the propeller regime of disc accretion in which the angular velocity of the stellar magnetosphere exceeds that of the inner disc. In these systems, the stellar magnetosphere acts as a centrifugal barrier and inhibits matter accretion onto the rapidly rotating star. Instead, the matter accreting through the disc accumulates at the disc-magnetosphere interface where it picks up angular momentum and is ejected from the system as a wide-angled outflow which gradually collimates at larger distances from the star. If the ejection rate is lower than the accretion rate, the matter will accumulate at the boundary faster than it can be ejected; in this case, accretion onto the star proceeds through an episodic accretion instability in which the episodes of matter accumulation are followed by a brief episode of simultaneous ejection and accretion of matter onto the star. In addition to the matter dominated wind component, the propeller outflow also exhibits a well-collimated, magnetically-dominated Poynting jet which transports energy and angular momentum away from the star. The propeller mechanism may explain some of the weakly-collimated jets and winds observed around some T Tauri stars as well as the episodic variability present in their light curves. It may also explain some of the quasi-periodic variability observed in cataclysmic variables, millisecond pulsars and other magnetized stars.
\end{abstract}

\section{Introduction}

An accreting magnetized star in spin equilibrium with its disc will enter the "propeller regime" of accretion if the accretion rate in the disc declines on a timescale shorter than the star's spin reequilibration timescale. When the accretion rate declines, the magnetosphere of the star expands outward due to the decrease in the ram pressure from the disc. In this new configuration, the angular velocity of the expanded stellar magnetosphere (which was previously in equilibrium) exceeds the Keplerian angular velocity of the matter in the inner disc, creating a centrifugal barrier at the disc-magnetosphere boundary. The propeller regime is characterized by three main features: firstly, the accretion of matter from the disc onto the star is inhibited by the centrifugal barrier; secondly, the centrifugal force may accelerate matter into an outflow; and lastly, the rapidly rotating star spins down due to the spindown torque exerted on the star.

The propeller effect is expected to be present in all types of accreting magnetized stars, ranging from newly formed protostars to accreting millisecond pulsars. It has been studied analytically using

\footnotetext{
ae-mail: pslii@astro.cornell.edu

This is an Open Access article distributed under the terms of the Creative Commons Attribution License 2.0, which permits unrestricted use, distribution, and reproduction in any medium, provided the original work is properly cited.
} 

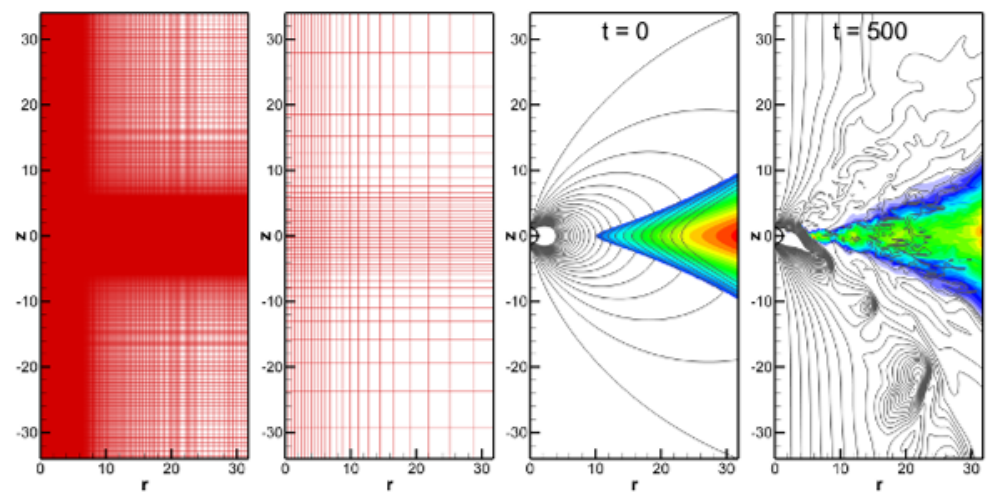

Figure 1. The Grid. From left to right: (1) grid used in simulations, (2) same grid but with $1 / 10$ th of the grid lines shown for clarity; (3) initial distribution of density (background) and magnetic flux (lines); (4) same but for time $t=500$.

using magnetohydrodynamics [MHD, e.g. 1-6] as well as numerically in MHD simulations [7-12]. Here, we summarize the results of a recent numerical study of the propeller regime utilizing a highresolution magnetohydrodynamics (MHD) code in cylindrical coordinates.

\section{The numerical model}

To study the propeller regime of accretion, we utilize a Godunov-type numerical method with a fivewave Harten, Lax, and van Leer (HLL) Riemann solver similar to the HLLD solver developed in [13]. Our method solves an equation for the entropy instead of the full energy equation. We ensure that the magnetic fields are divergence-free by introducing the $\phi$-component of the magnetic field potential which is calculated using the constrained transport scheme proposed by [14]. No viscosity or diffusivity terms have been included in the MHD equations and hence we investigate only accretion driven by the MHD turbulence associated with the magnetorotational instability (MRI) [see tests and other details in 15].

The code utilizes an axisymmetric grid in cylindrical $(r, z)$ coordinates with mesh compression towards the disc and towards the $z$-axis (see Fig. 1, left panel). In the models presented here, we use a non-uniform grid with dimension $250 \times 432$ cells corresponding to a grid that is 33 by 70 stellar radii in size ( $0.31 \mathrm{AU}$ by $0.65 \mathrm{AU}$ if $\left.R_{*}=2 R_{\odot}\right)$. The initial conditions for the hydrodynamic variables are similar to those taken in our previous works [e.g. 15, 16] where the initial density and entropy distributions have been calculated from the force-balance of different forces which include gravity, centrifugal and pressure forces. The disc is initially dense and cold while the corona is about 1000 hotter and of lower density. Initially, the disc is threaded with the dipole magnetic field of the star in addition to a weak "tapered" poloidal field inside the disc which helps to initialize the MRI (see third panel in Fig. 1).

\section{Magnetized stars in the propeller regime}

The propeller regime of accretion is characterized by three main features: the inhibition of accretion, the emergence of centrifugally-driven outflows, and the spindown of the propelling star. In this section we discuss each of these three phenomena in turn. 


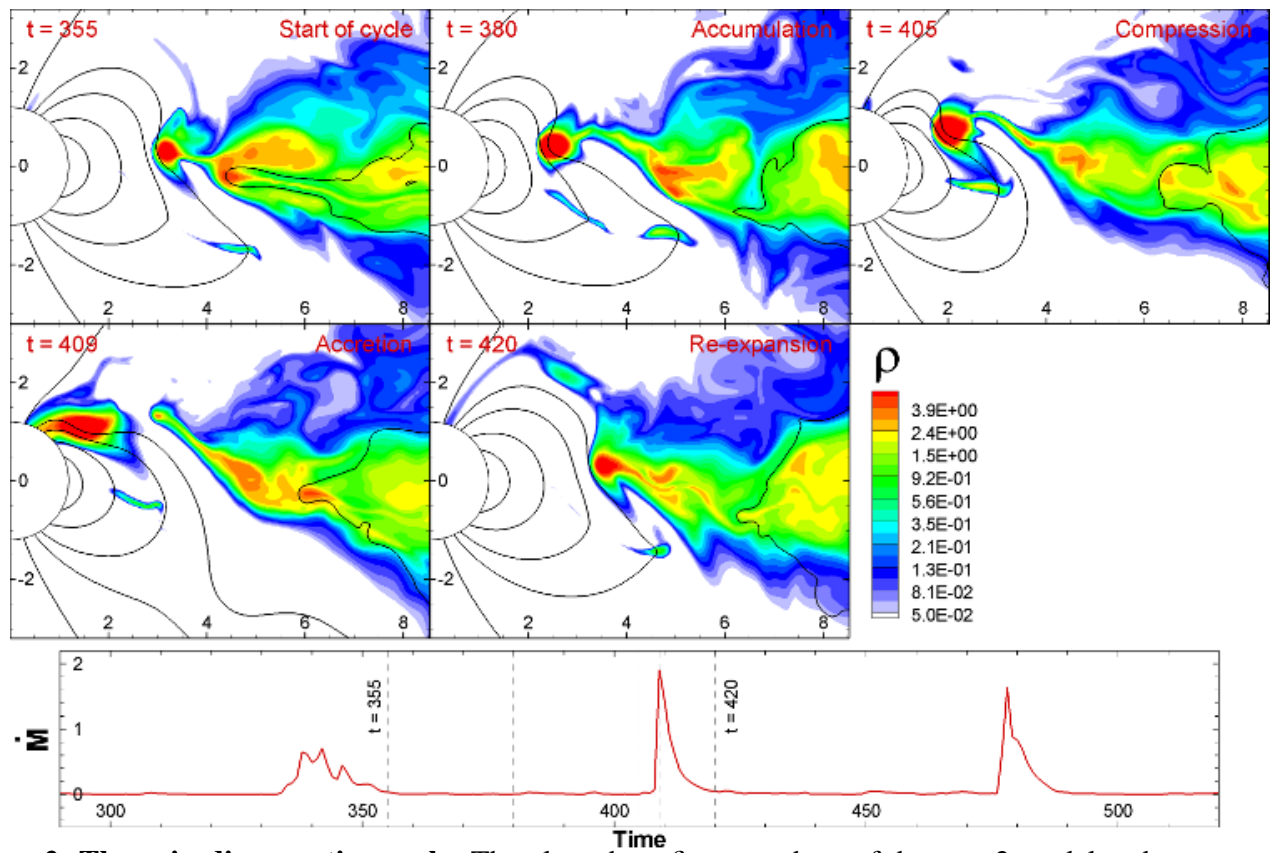

Figure 2. The episodic accretion cycle. The plots show five snapshots of the $r_{\mathrm{cor}}=2$ model and trace one cycle of the episodic accretion cycle. The color contours show the matter density $\rho$, while the lines trace the poloidal magnetic field lines. The bottom panel shows the mass accretion rate onto the star $\dot{M}_{*}$ along with vertical dotted lines which correspond to the snapshots shown in the top panels.

\subsection{Episodic accretion onto the star}

The inner disc dynamics are largely determined by the interaction between the disc and magnetic fields at the disc-magnetosphere boundary. In the propeller regime, the centrifugal force plays the dominant role in this interaction. The centrifugal barrier inhibits accretion onto the rapidly rotating star; however, accretion can proceed in episodic bursts through the episodic accretion instability developed in [17-19]. The episodic accretion instability proceeds as follows:

1. Accumulation: The matter accreting through the disk is blocked from accreting onto the star by the strong centrifugal barrier. Instead, it accumulates at the disc-magnetosphere boundary, gradually compressing the stellar magnetosphere inwards towards the star.

2. Compression and accretion: The matter continues to compress the magnetosphere toward the star until the gravitational acceleration is larger than the centrifugal acceleration, at which point the matter accretes onto the star as a funnel flow.

3. Re-expansion: With the accumulated reservoir of matter depleted, the magnetosphere is no longer compressed and quickly re-expands out and shuts off the accretion, restarting the cycle anew.

Figure 2 shows one cycle of the episodic accretion instability in a propeller model with $r_{\text {cor }}=2$ (corresponding to a rotation period of 6.6 days for a typical cTTs). The timescale between each accretion event is dependent on the disc accretion rate as well as the spin period of the star. This instability results in a "spiky" accretion rate in which any accretion onto the star proceeds in quasi-cyclic bursts with frequencies of up to a hundred dynamical timescales (see bottom panel of Figure 2). 

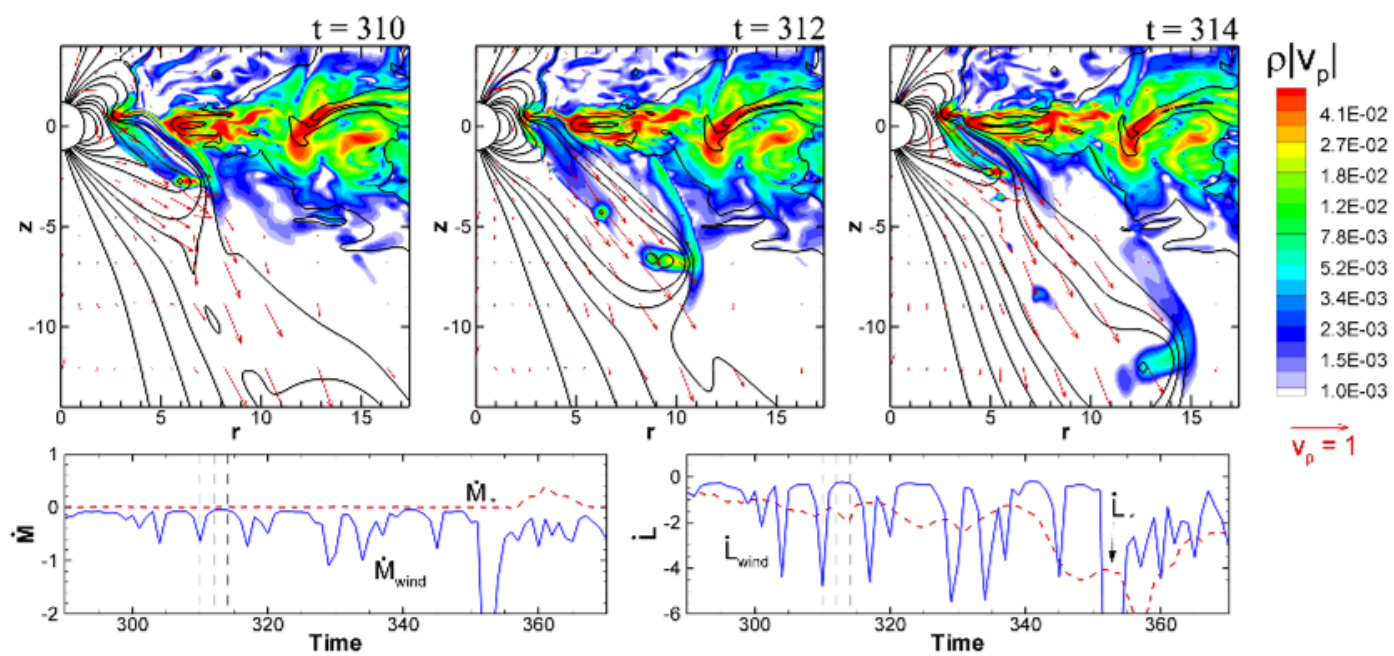

Figure 3. The non-accreting outflow mode. Top panels: three snapshots of the $r_{\text {cor }}=1.3$ model showing the plasmoids launched during the non-accreting phase of the episodic accretion cycle. The color contours show the matter flux density $\rho v_{\mathrm{p}}$ with the magnetic field lines and velocity vectors superimposed. Bottom panels: matter and angular momentum fluxes onto the star and into the wind. The vertical lines denote the times corresponding to the three snapshots in the top panels.

\subsection{Propeller-driven outflows}

The rapid rotation of the stellar magnetosphere can result in an centrifugally-driven outflow originating at the disc-magnetosphere boundary [3]. In order to investigate these outflows, we study the case of a star accreting in the strong propeller regime with a corotation radius $r_{\text {cor }}=1.3$ (corresponding to a cTTs with a short 3.4 day period). In the strong propeller regime, the centrifugal force at the disc-magnetosphere boundary is sufficiently large to accelerate the matter in the inner disc into an outflow which leaves the system. In contrast, in the weak propeller regime where $r_{\text {mag }}<1.3 r_{\text {cor }}$, the centrifugal force at the disc-magnetosphere boundary is insufficient to accelerate the matter past the local escape velocity, resulting in a trapped disc in which the matter is trapped or continuously recycled instead of being ejected [20]. We consider the former case.

The propelling stars which we study exhibit two modes of outflows. The primary mode is a continuous, non-stationary outflow driven in the non-accreting (accumulation) phase of the episodic accretion cycle. Figure 3 shows three time snapshots of the outflow in the $r_{\text {cor }}=1.3$ model. The primary outflow mode proceeds in accordance with the cyclic inflation-reconnection mechanism first described by [21]. During the accumulation phase of the episodic accretion cycle, some of the accumulated matter diffuses into the outer magnetosphere. There, the differential rotation between the star and matter induces helicity in the field lines and inflates the magnetosphere, providing a path along which the matter can flow $(\mathrm{t}=310)$. The matter is accelerated away along these opened field lines by the centrifugal force near the star $(t=312)$. As the fields continue to inflate, the magnetic tension eventually causes the field lines at the base of the outflow to reconnect, cutting off the outflow $(\mathrm{t}=$ 314 ) and restarting the cycle. As evidenced by the bottom panels in Fig. 3, the outflow in the $r_{\text {cor }}=1.3$ model results in the ejection of discrete plasmoids, similar to the clumpy winds observed in previous studies of propeller outflows [e.g 9, 10,12]. The matter is ejected with a wide half-opening angle of $\sim 45^{\circ}$, as measured at the base of the propeller wind. 

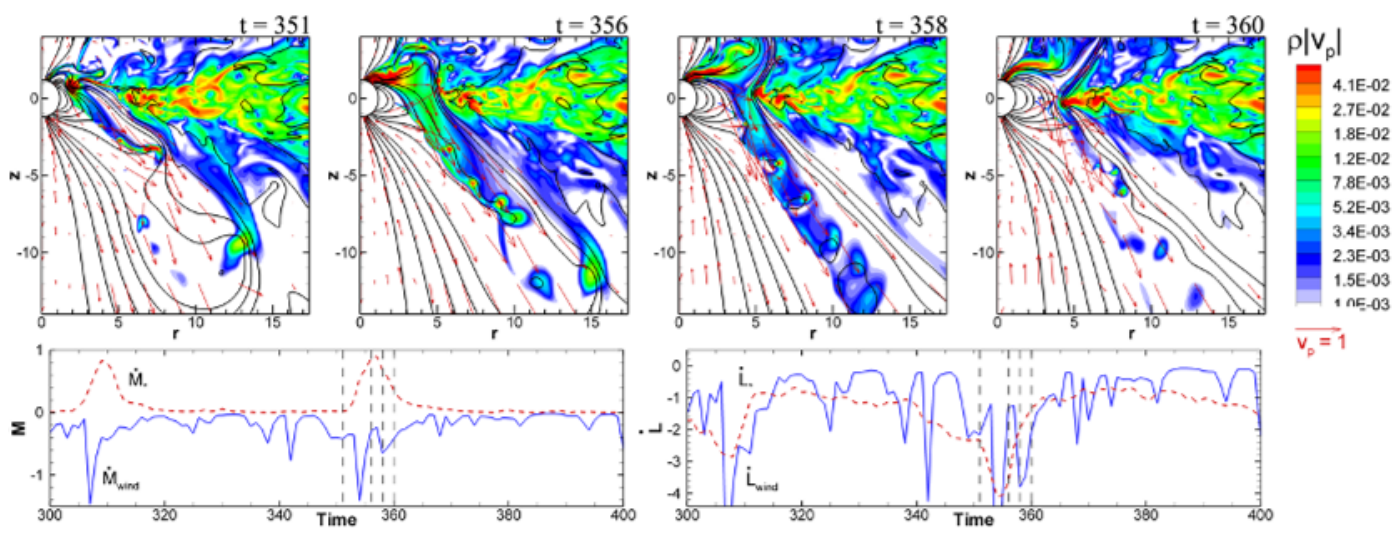

Figure 4. The accreting outflow mode. Top panels: four time snapshots of the propeller outflow during the burst of accretion at the end of an episodic accretion cycle. A large amount of matter is ejected into the outflow when the accumulated matter accretes onto the star. Bottom panels: the mass and angular momentum fluxes onto the star and into the wind. The vertical dashed lines indicate the times corresponding to the four snapshots above.

The second outflow mode is a transient outburst associated with the accretion event at the end of the episodic accretion cycle. Fig. 4 shows this outflow in a series of four snapshots from the same $r_{\text {cor }}=1.3$ model as above. At $\mathrm{t}=351$, the magnetosphere is approaching the end of an episodic accretion cycle: the matter reservoir at the disc-magnetosphere interface has compressed the field lines inward enough for accretion to proceed onto the star. As it approaches the star, the matter deflects the magnetosphere to one side and forms a transient funnel flow $(\mathrm{t}=356)$. At the same time, some of the infalling matter penetrates into the outer magnetosphere and picks up angular momentum from the magnetic field lines. As it is accelerated away, it inflates the magnetosphere, providing a path for the rest of the matter to flow away $(t=358)$. Once the reservoir of matter compressing the magnetosphere is depleted by the accretion and ejection, the magnetosphere re-expands outward and reverts the outflow back to the initial non-accreting state. As shown in the bottom panels of 4, the simultaneous accretion-ejection event results in a brief rise in the outflow rate. For this stronger outflow, we measure half-opening angles ranging from $20-40^{\circ}$ at the simulation boundary (compared to the wider $\sim 45^{\circ}$ wind in the non-accreting phase).

One key feature of these outflows is that they are predominately one-sided. Initially, the magnetic fields are equatorially symmetric. However, as soon as the matter from the disc reaches the magnetosphere, the magnetosphere is deflected to one side and an asymmetry is induced in the magnetic fields. This asymmetry helps to drive the one-sided outflows as it provides a preferential direction for the matter accretion and ejection [see 22, for more on one-sided outflows].

\subsection{The Poynting jet and propeller spindown}

The coupling between the star and disc alone is insufficient to account for the entirety of the spindown torque on the star [see 12]. Rather, most of the stellar angular momentum flows away in the propeller-driven outflow and in the opened field lines connecting the star to the slower rotating corona (i.e. the Poynting jet). Initially, the magnetic configuration of the magnetic field is that of an aligned dipole. However, as the star rotates, the field lines near the stellar poles (which are coupled to the disc) inflate upwards, forming the magnetic tower which comprises the Poynting jet. In the jet, the 

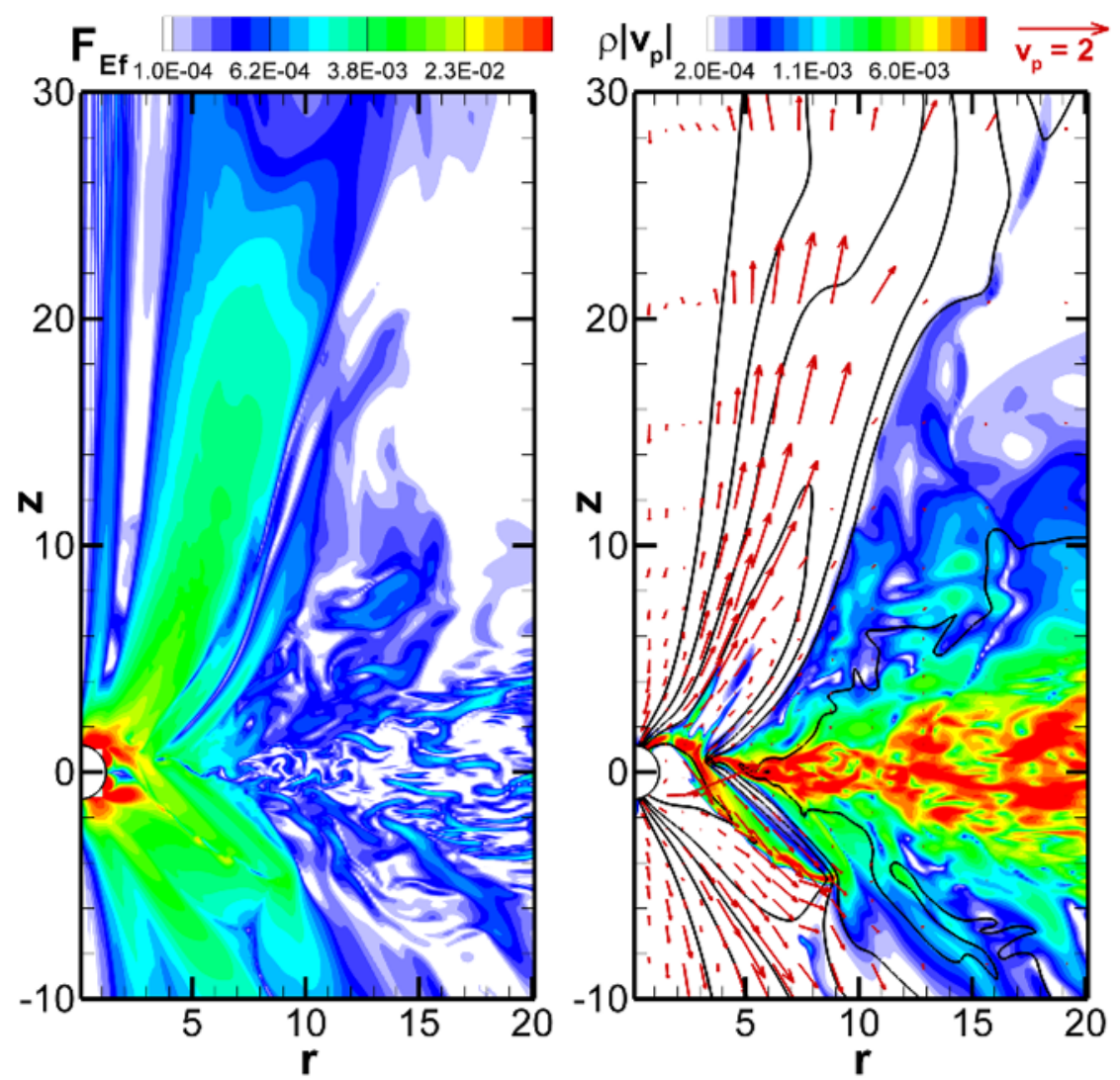

Figure 5. Energy flux density. Left panel: the color contours show the energy flux density in the magnetic fields. Right panel: matter flux density with poloidal field lines and velocity vectors overplotted. These plots show the $r_{\text {cor }}=1.3$ model at $\mathrm{t}=400$.

magnetic pressure gradient accelerates the low-density matter away from the star while the hoop stress collimates the outflow $[23,24]$. In Figure 5, we plot the magnetic energy flux density associated with the Poynting jet at $\mathrm{t}=400$

$$
\mathbf{F}_{\mathrm{Ef}}=\frac{c}{4 \pi} \mathbf{E} \times \mathbf{B}=\frac{|\mathbf{B}|^{2} \mathbf{v}_{\mathbf{p}}-(\mathbf{B} \cdot \mathbf{v}) \mathbf{B}_{\mathbf{p}}}{4 \pi} .
$$

Inside the magnetic tower, the matter density is low but there is nonetheless significant energy outflow through the magnetic field lines. These magnetic fields help to remove both energy and angular momentum from the rapidly rotating star. The inner, strongly magnetically-dominated regions of the jet exhibit stronger collimation than the outer regions where there is more matter. For the Poynting jet, we measure narrow half-opening angles of $5-15^{\circ}$ at the edge of the simulation region.

We estimate the long-term spin-down rate of the star by time-averaging the angular momentum flux out of the stellar surface and determine a characteristic spin-down timescale $t_{\mathrm{sp}}=L_{*} / \dot{L}$ of about $1 \mathrm{Myr}$ for the fast rotating $r_{\text {cor }}=1.3$ model (assuming parameters for a typical cTTs). This spin-down timescale is roughly independent of spin period; this is because the faster rotators have larger spindown rates, but also higher values of initial angular momentum. 
Physics at the Magnetospheric Boundary

\section{Conclusion}

In the propeller regime of accretion, the centrifugal barrier at the disc-magnetosphere boundary dominates the inner-disc dynamics. Propelling stars undergo an episodic accumulation-accretion instability which results in unstable accretion onto the star. In very rapidly rotating propellers, the strong centrifugal force may drive outflows from disc-magnetosphere boundary. This outflow occurs in two modes corresponding to the accumulation and accretion phases of the episodic accretion cycle. The rapidly rotating star also drives a magnetically-dominated Poynting jet which contributes to the spindown of the star. In our models, we estimate spin-down timescales of roughly 1 Myr for a typical cTTs.

\section{Acknowledgments}

We thank G. Ustyugova and A. Koldoba for helpful discussions and their support of the code used to perform this research.

\section{References}

[1] R.E. Davies, A.C. Fabian, J.E. Pringle, MNRAS 186, 779 (1979)

[2] J. Li, D.T. Wickramasinghe, Disc Accretion Onto Magnetic Stars: Slow Rotator and Propeller, in IAU Colloq. 163: Accretion Phenomena and Related Outflows, edited by D. T. Wickramasinghe, G. V. Bicknell, \& L. Ferrario (1997), Vol. 121 of Astronomical Society of the Pacific Conference Series, p. 241

[3] R.V.E. Lovelace, M.M. Romanova, G.S. Bisnovatyi-Kogan, ApJ 514, 368 (1999)

[4] N.R. Ikhsanov, A\&A 381, L61 (2002)

[5] S.A. Rappaport, J.M. Fregeau, H. Spruit, ApJ 606, 436 (2004)

[6] K.Y. Ekşi, L. Hernquist, R. Narayan, ApJL 623, L41 (2005)

[7] Y.M. Wang, J.A. Robertson, A\&A 151, 361 (1985)

[8] M.M. Romanova, G.V. Ustyugova, A.V. Koldoba, R.V.E. Lovelace, ApJ 610, 920 (2004)

[9] M.M. Romanova, G.V. Ustyugova, A.V. Koldoba, R.V.E. Lovelace, ApJL 635, L165 (2005)

[10] G.V. Ustyugova, A.V. Koldoba, M.M. Romanova, R.V.E. Lovelace, ApJ 646, 304 (2006)

[11] M.M. Romanova, G.V. Ustyugova, A.V. Koldoba, R.V.E. Lovelace, MNRAS 399, 1802 (2009)

[12] C. Zanni, J. Ferreira, A\&A 550, A99 (2013)

[13] T. Miyoshi, K. Kusano, Journal of Computational Physics 208, 315 (2005)

[14] T.A. Gardiner, J.M. Stone, Journal of Computational Physics 205, 509 (2005)

[15] M.M. Romanova, G.V. Ustyugova, A.V. Koldoba, R.V.E. Lovelace, MNRAS 416, 416 (2011)

[16] M.M. Romanova, G.V. Ustyugova, A.V. Koldoba, R.V.E. Lovelace, ApJ 578, 420 (2002)

[17] R.A. Siuniaev, N.I. Shakura, Pisma v Astronomicheskii Zhurnal 3, 262 (1977)

[18] H.C. Spruit, R.E. Taam, ApJ 402, 593 (1993)

[19] C.R. D’Angelo, H.C. Spruit, MNRAS 406, 1208 (2010)

[20] C.R. D'Angelo, H.C. Spruit, MNRAS 420, 416 (2012)

[21] A.P. Goodson, R.M. Winglee, K. Boehm, ApJ 489, 199 (1997)

[22] R.V.E. Lovelace, M.M. Romanova, G.V. Ustyugova, A.V. Koldoba, MNRAS 408, 2083 (2010)

[23] G.V. Ustyugova, R.V.E. Lovelace, M.M. Romanova, H. Li, S.A. Colgate, ApJL 541, L21 (2000)

[24] R.V.E. Lovelace, H. Li, A.V. Koldoba, G.V. Ustyugova, M.M. Romanova, ApJ 572, 445 (2002) 
